Article

\title{
Distinct Metabolome Changes during Seed Germination of Lettuce (Lactuca sativa L.) in Response to Thermal Stress as Revealed by Untargeted Metabolomics Analysis
}

\author{
Shiwei Wei ${ }^{1,+}{ }^{\dagger}$ Xiao Yang ${ }^{2,3, \dagger}$, Guotao Huo ${ }^{1}$, Guojun Ge ${ }^{1}$, Hongyan Liu ${ }^{1}$, Lijun Luo ${ }^{1}$, \\ Jinguo $\mathrm{Hu}^{4}$, Danfeng Huang ${ }^{3, *}$ and Ping Long ${ }^{1, *}$ \\ 1 Shanghai Agrobiological Gene Center, Shanghai 201106, China; wsw@sagc.org.cn (S.W.); \\ hgt@sagc.org.cn (G.H.); ggj@sagc.org.cn (G.G.); lhy@sagc.org.cn (H.L.); lijun@sagc.org.cn (L.L.) \\ 2 Institute of Urban Agriculture, Chinese Academy of Agricultural Sciences, Chengdu National Agricultural \\ Science and Technology Center, Chengdu 610213, China; yangxiao@caas.cn \\ 3 School of Agriculture and Biology, Shanghai Jiao Tong University, Shanghai 200240, China \\ 4 US Department of Agriculture, Western Regional Plant Introduction Station, Washington State University, \\ Pullman, WA 99164, USA; jinguo.hu@usda.gov \\ * Correspondence: hdf@sjtu.edu.cn (D.H.); lp@sagc.org.cn (P.L.) \\ + These authors contributed equally to this work.
}

Received: 19 December 2019; Accepted: 18 February 2020; Published: 21 February 2020

\begin{abstract}
Temperature strongly influences lettuce (Lactuca sativa L.) seed germination. Different lettuce genotypes respond differently to higher temperatures or thermal stress. In this study, we evaluated the germination performance of 304 lettuce accessions incubated at three temperature settings, $21^{\circ} \mathrm{C}$, $28^{\circ} \mathrm{C}$ and $35^{\circ} \mathrm{C}$, respectively, for $40 \mathrm{~h}$. At $21^{\circ} \mathrm{C}$, seeds of all 304 accessions germinated with very well an average germination percentage of $87.72 \%$; at $28{ }^{\circ} \mathrm{C}$, the average germination percentage dropped to $42.84 \%$ and at $35^{\circ} \mathrm{C}$, the germination decreased to $1.01 \%$. Then, we investigated changes in metabolic profiles of lettuce seed response to thermal stress using an untargeted metabolomics approach. Results suggested that seeds of thermal-sensitive and thermal-tolerant cultivars employed different metabolic strategies in response to thermal stress during germination. Thermal-sensitive buds accumulated more significant amounts of organic acids, amino acids, sugars, sterols, phenolic compounds and terpenoids compared to thermal-tolerant buds at $21^{\circ} \mathrm{C}$. Thermal-tolerant lettuce cultivar accumulated higher concentrations of amino acids, organic acids, sugars, sesquiterpene lactones, sterols, and fatty acids derivatives during the germination at $35^{\circ} \mathrm{C}$ compared to germinated at $21^{\circ} \mathrm{C}$. This investigation paves the way to link the metabolomics to other external and internal factors affecting lettuce seed germination under thermal stress.
\end{abstract}

Keywords: lettuce; germination; thermoinhibition; thermal stress; metabolomics

\section{Introduction}

Thermoinhibition refers to the phenomenon in which viable seeds failed to germinate at high temperature and it is recognized as an adaptive regeneration strategy for the cool-season crop to prevent seeds from germination at inappropriate high-temperature particularly in summer that may hamper the growth and development of the young plant [1]. In agricultural production, thermoinhibition limits the time and area for the production of various crops, including vegetables [2]. For example, most of the cultivars of the worldwide consumed vegetable crop, lettuce, exhibit seed thermoinhibition [3]. The optimal temperature for lettuce seed germination ranges from 18 to $21^{\circ} \mathrm{C}$ [4]. The lettuce seed 
germination is greatly hampered by relatively high temperatures $\left(>25^{\circ} \mathrm{C}\right)$, such as in warm-season or in topical zones, when or where the temperature is always higher than the upper-temperature limitation of lettuce germination $[2,3,5,6]$. Therefore, understanding the mechanism of lettuce seed germination and thermoinhibition will greatly increase lettuce breeding efficiency in producing thermal tolerance cultivars to ensure good germination during warm season planting.

Due to its significance in both basic study and applied crop production, this fascinating phenomenon of thermoinhibition has been investigated for a long time. In the 1960s, researchers found that external factors such as light [7] and internal factors such as plant hormones [1,8] could alter seed germination under higher temperatures. ABA-related genes were more highly expressed when germination was inhibited, and GA- and ethylene-related genes were more highly expressed when germination was permitted [9]. In recent years, contemporary approaches, including genome sequencing, transcriptomics and proteomics have been deployed to gain insights of the complex nature of lettuce seed germination in response to heat stress $[2,9,10]$. A few key genes have been identified to be associated with the thermoinhibition of lettuce seeds. For instance, the upregulation of abscisic acid biosynthetic enzyme gene 9-cis-EPOXYCAROTENOID DIOXYGENASE4 results in germination thermoinhibition of lettuce seeds [3]. Considering that all thermoinhibition-affecting factors identified thus far will alter gene expression leading to synthesize or decompose certain metabolites, we intend to tackle this phenomenon from a different angle, using the metabolomic approach to gain information towards a better understanding of its mechanism.

Metabolomics has been widely employed to establish the connections between genotype and phenotype and is capable of identification and quantification of different classes of metabolites simultaneously and unbiasedly [11,12]. Non-targeted metabolomics approaches for lettuce have been developed and optimized for various research objectives previously. For instance, Garcia et al. using LC-MS based untargeted metabolomics approach compared differences of metabolome between two romaine lettuces after cutting and revealed that phenolic, terpenoid and lipid metabolites could explain the browning process in lettuce and be used as biomarkers to predict browning of fresh-cut lettuce $[13,14]$. An integrated GC-MS and LC-MS based untargeted metabolomic analysis was launched to compare the metabolic difference occurred in primary and secondary metabolism of lettuce hydroponically cultivated in organic and inorganic nitrogen culture, results suggested that organic nitrogen promoted the accumulation of glycosylated flavonoids, ascorbic acid and amino acids, but inhibited the content of phenolic acids and intermediates of the Krebs Cycle when compared to those lettuce cultivated under nitrate solution [15]. Yang et al. used combined GC $\times$ GC-TOF/MS and UPLC-IMS-QTOF/MS non-targeted metabolomic strategy to detect and relatively quantify metabolites in leafy and head lettuce cultivars, and found that 16 metabolites including phenolic acid derivatives, glycosylated flavonoids, and one iridoid were present at significantly different levels in leaf and head type lettuces [16]. However, to the best of our knowledge, using a large-scale untargeted metabolomics analysis to compare the metabolic diversity of lettuce seed germination and thermoinhibition has not been reported.

In this study, we evaluated the germination percentage of 274 lettuce accessions from Shanghai Agricultural Gene Center (SAGC) and 30 commercial cultivars purchased from the local market after being incubated for $40 \mathrm{~h}$ at $21^{\circ} \mathrm{C}, 28^{\circ} \mathrm{C}$ and $35^{\circ} \mathrm{C}$, respectively, in a germination chamber. Based on the obtained results, two lettuce accessions, one thermotolerant entry ('s15k0106', with the highest germination percentage at $35{ }^{\circ} \mathrm{C}$ ) and one thermosensitive entry ('s13k0062', zero germination at $35^{\circ} \mathrm{C}$ ) were selected for further study using a metabolomics approach. We combined gas chromatography-mass spectrometry (GC/MS) and ultra-performance liquid chromatography-ion mobility spectrometry quadrupole time-of-flight mass spectrometry (UPLC-IMS-QTOF/MS) platforms to reveal the primary and secondary metabolites among the seed samples of the two selected accessions of dry seeds (seeds without germination tests), germinated seeds and ungerminated seeds at two temperature settings, $21^{\circ} \mathrm{C}$ and $35^{\circ} \mathrm{C}$, respectively. We anticipate that this investigation will detect 
differences among the seed samples of different genotypes under different treatments and gain insight regarding the metabolic mechanism of lettuce seed thermoinhibition.

\section{Results}

\subsection{Country of Origin, Seed Coat Color and Seed Weight of the Lettuce Germplasm Used in The Current Study}

The 304 lettuce accessions used in this study were collected from the 34 countries (Supplemental Table S1). A large proportion (29.28\%) of the germplasm was obtained from the Netherlands, followed by Turkey (17.76\%), United States (13.49), China (11.51\%) and another 28 countries (22.04\%). For seed coat color, 194 accessions are white and 110 black. The average 100-grain weight for all 304 accessions was $0.118 \mathrm{~g}$. Among all accessions, S14K0333 had the highest 100-grain weight of $0.183 \mathrm{~g}$, and S15K050 had the lowest 100-grain weight of $0.066 \mathrm{~g}$ (Supplemental Table S1).

\subsection{Evaluation of Seed Germination under Different Temperatures}

Lettuce genotypes differ greatly in their ability to germinate at different temperatures, as determined by the rates of germination. We observed a high average germination rate of $87.72 \%$ at $21{ }^{\circ} \mathrm{C}$ for all 304 accessions; however, the germination rate decreased to $42.84 \%$ at $28^{\circ} \mathrm{C}$ and $1.01 \%$ at $35^{\circ} \mathrm{C}$, respectively; and these differences are statistically significant $(p<0.01)$, indicating lettuce seeds are sensitive to high-temperature stress (Figure 1). Sixteen accessions had high percentage germination of $>90 \%$ at $28{ }^{\circ} \mathrm{C}$, with s15k091 having the highest percentage germination $(98.33 \%)$. Five accessions had a high percentage of germination $(>10 \%)$ at $35^{\circ} \mathrm{C}$. s15k106 showed the highest percentage germination at $35^{\circ} \mathrm{C}(39.67 \%)$, with corresponding germination percentages of $74.66 \%$ at $28^{\circ} \mathrm{C}$ and $76.00 \%$ at $21^{\circ} \mathrm{C}$ (Supplementary Table S1).

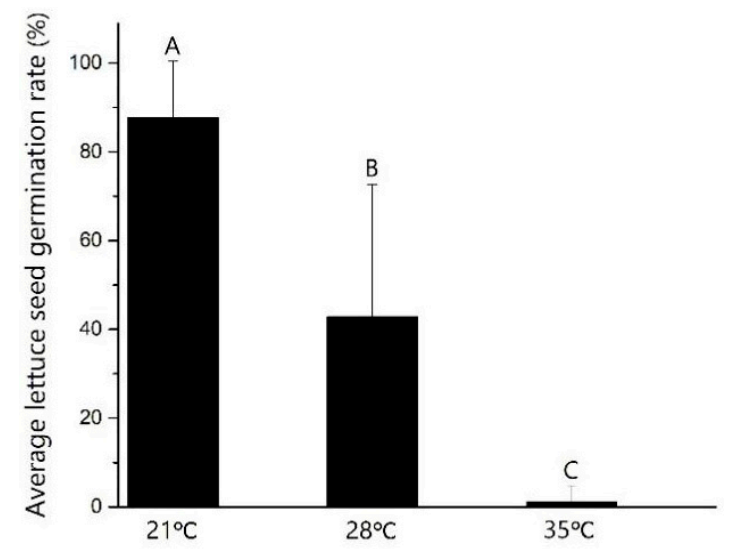

Figure 1. Average lettuce seed germination rate at different temperatures (\%). The average lettuce seed germination rate of all 304 accessions was $87.72 \%$ at $21{ }^{\circ} \mathrm{C}, 42.84 \%$ at $28{ }^{\circ} \mathrm{C}$ and $1.01 \%$ at $35{ }^{\circ} \mathrm{C}$, respectively. Different capital letters indicate significant differences, $p<0.01$; LSD analysis $(n=304)$.

\subsection{The Seed Color, Weight and Origin Region Influence on Germination Rates of Lettuce under Heat Stress}

There was no relationship between dark and white seed coats on germination rates at $28^{\circ} \mathrm{C}(46.43 \%$ vs. $51.12 \%$, respectively; $p=0.243)$ or $35^{\circ} \mathrm{C}(1.16 \%$ vs. $1.23 \%$, respectively; $p=0.753)$. In addition, there were no relationships between 100-grain weight and seed color $(0.12 \mathrm{~g}$ vs. $0.12 \mathrm{~g}$ for dark and white seeds, respectively; $p=0.267$ ) or 100 -grain weight and seed source $(0.12 \mathrm{~g}$ vs. $0.11 \mathrm{~g}$ for accessions and cultivars, respectively; $p=0.157)$. However, there was a negative relationship between relative percent germination and 100 -grain weight at $28^{\circ} \mathrm{C}\left(\mathrm{R}^{2}=-0.397, p<0.01\right)$, but not at $35^{\circ} \mathrm{C}$.

\subsection{Metabolic Profiles of Lettuce Seeds}

Seeds of two cultivars, a thermosensitive variety (s13k0062) and a thermotolerant variety (s15k0106), were selected to investigate the metabolic mechanisms of seed germination at high temperature. 
A non-targeted metabolomics strategy combining GC/MS and UPLC-IMS-QTOF-MS was used to simultaneously detect and enable relative quantification of primary and secondary metabolites of lettuce [15]. Stable intensities were detected for the internal standards (L-2-chlorophenylalanine for GC/MS and EGCG for UPLC-IMS-QTOF/MS) in each sample. In addition, the drift retention times for the same internal standard peaks in different runs were less than $0.02 \mathrm{~min}$ in both GC/MS and UPLC-IMS-QTOF/MS analysis. Moreover, the Quality control (QC) samples clustered together in the center of the PCA scores in UPLC-IMS-QTOF/MS analysis (Supplemental Figure S1). Collectively, these results suggest the data acquisition was reproducible and robust. Overall, we extracted 998 features from the matrix in GC/MS analysis, and 8127 features were extracted from the UPLC-IMS-QTOF/MS.

\subsection{Identification of Differential Metabolites}

We performed unsupervised PCA analysis to determine the variations in metabolites between different cultivars and at different germination temperatures. A five-principle component-based robustness PCA model with a $\mathrm{Q}^{2}$ (cum) value of 0.491 was established. PC1 and PC2 explained 53.6\% of the total variation, and clear separation was observed among the six different groups; the optimal germination temperature treatments (C106 and C62) were distributed far from the center of the coordinate axis (Figure 2). To identify potential variables, we screened the differential metabolites by comparing the fold change $(>2), p$-value $(<0.01)$, and variable importance in projection (VIP) score $(>1.0)$ of the metabolites in each pair of comparisons and then identified these differential metabolites using online databases and our in-house database.

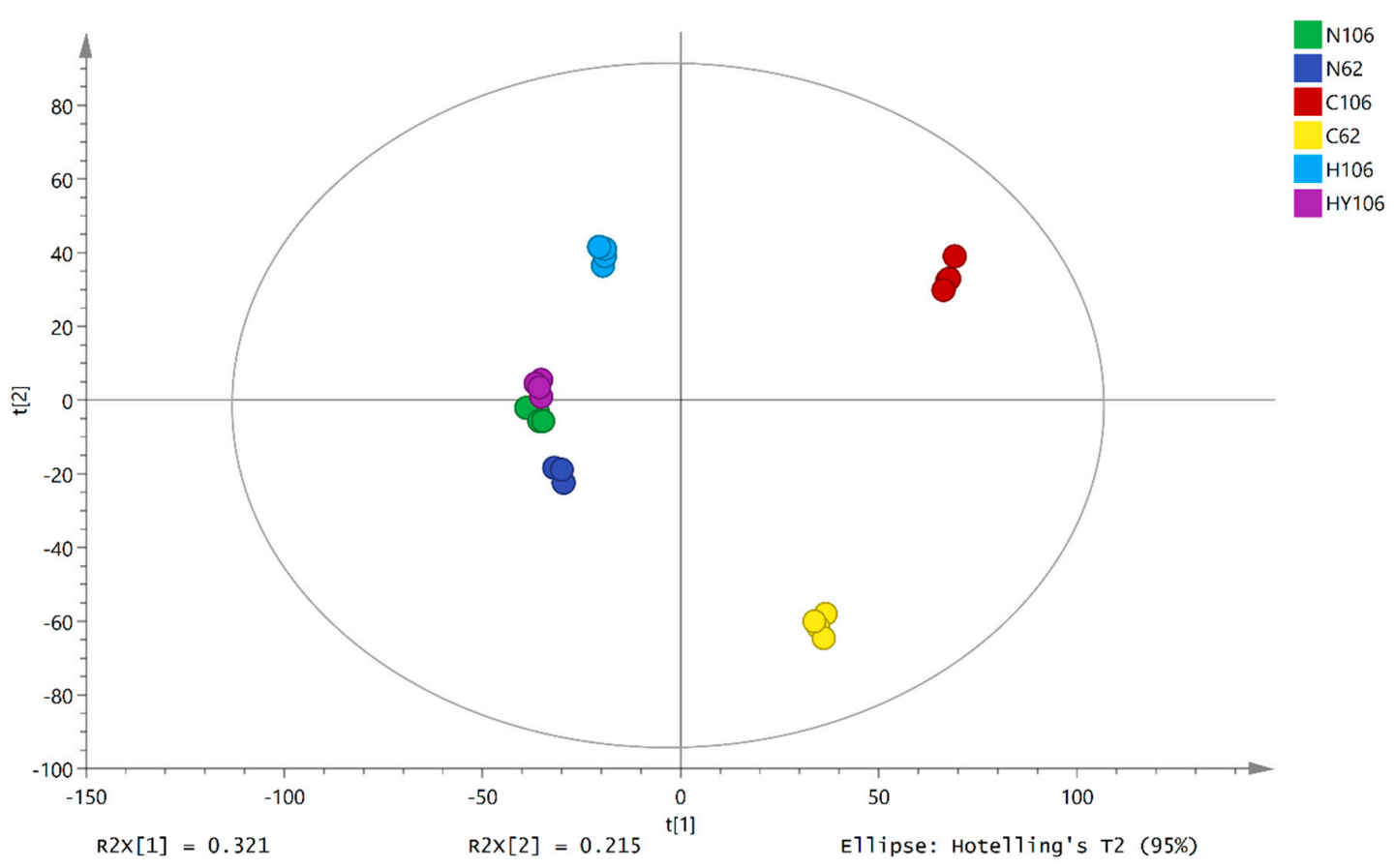

Figure 2. PCA score plots for different lettuce seed metabolomes. A five principle component-based PCA model $\left(\mathrm{R}^{2} \mathrm{X}_{(\mathrm{cum})}=0.774, \mathrm{Q}^{2}\right.$ (cum) $\left.=0.49\right)$ for GC/MS and UPLC-IMS-QTOF/MS data was obtained; the PCA analysis separated six different groups (N62: S13K0062 dry seeds; N106: S15K106 dry seeds; C106: buds of S15k0106 seeds germinated at $21^{\circ} \mathrm{C}$; C62: buds of S13k0062 seeds germinated at $21{ }^{\circ} \mathrm{C}$; H106: S15K106 seeds fail to germination at $35^{\circ} \mathrm{C}$; HY106: buds of S15k0106 seeds germinated at $35{ }^{\circ} \mathrm{C}$ ) and revealed differences in the relative content of metabolites present in different groups.

2.6. Metabolite Differences between Thermo-Tolerant and Thermo-Sensitive Seeds

A total of 867 metabolites were marked as differential metabolite candidates in the comparison between the thermotolerant seeds (N106) and thermosensitive seeds (N62); 11 of these metabolites were putatively annotated (Supplemental Tables S2-S4 and Figure S2A). N106 seeds had higher 
concentrations of the unsaturated long-chain fatty acids, including 9,12-octadecadienoic acid $(\mathrm{Z}, \mathrm{Z})$-, 9-octadecenoic acid (E)- and oleic acid (Z)-, and saturated long-chain fatty acid (arachidic acid), than N62; N62 seeds had higher accumulation of some organic acids (lactic acid, quininic acid), amino acids ( $\mathrm{L}$-methionine, L-ornithine, L-asparagine, L-methionine), as well as arabinitol and caffeoyl-hexose isomer 2 (Table 1 and Figure 3).

Table 1. Differential metabolites extracted from S15k0106 (N106) seeds and S13k0062 (N62) seeds.

\begin{tabular}{ccccc}
\hline No. & Compound & Log2 (FC N106/N62) & $p$-Value & VIP Value \\
\hline GC_95 & Lactic acid & -1.90 & $1.70 \times 10^{-5}$ & 1.26 \\
GC_361 & L-Methionine & -1.12 & $1.70 \times 10^{-5}$ & 1.26 \\
GC_453 & Asparagine & -1.75 & $3.58 \times 10^{-3}$ & 1.17 \\
GC_484 & Arabinitol & -1.03 & $3.31 \times 10^{-5}$ & 1.25 \\
GC_537 & L-Ornithine & -1.19 & $1.73 \times 10^{-5}$ & 1.26 \\
GC_554 & Quininic acid & -1.01 & $9.85 \times 10^{-5}$ & 1.25 \\
GC_686 & 9,12-Octadecadienoic acid (Z,Z)- & 1.00 & $2.69 \times 10^{-4}$ & 1.23 \\
GC_689 & 9-Octadecenoic acid, (E)- & 1.56 & $4.09 \times 10^{-5}$ & 1.25 \\
GC_692 & Oleic acid, (Z)- & 1.11 & $1.00 \times 10^{-3}$ & 1.21 \\
GC_786 & Arachidic acid & 1.06 & $9.46 \times 10^{-4}$ & 1.21 \\
LC_4594 & Caffeoyl-hexose 2 & -1.55 & $1.55 \times 10^{-5}$ & 1.26 \\
\hline
\end{tabular}

Note: Fold change of different metabolites between N106 and N62 is reported as Log2(FC N106/N62).

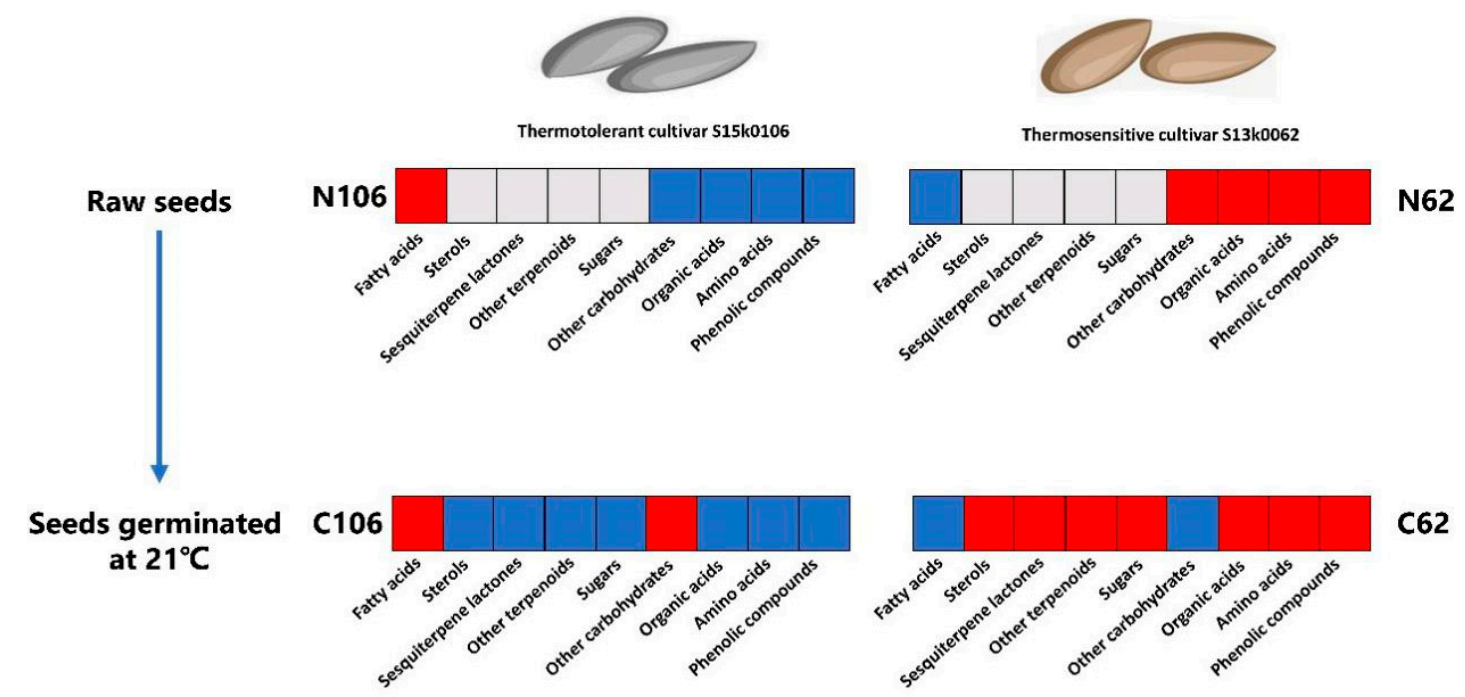

Figure 3. Differential metabolites detected between the thermotolerant and thermosensitive lettuce cultivars. Note: The heatmap indicates the differential metabolites extracted from raw S15k0106 (N106) and S13k0062 (N62) seeds and germinated buds of S15k0106 (C106) and S13k0062 (C62) seeds. A red box means that most of the metabolites in this class had higher relative abundances in a treatment than those in another in a pair of comparison (N106 vs. N62 or C106 vs. C62), while a blue box represents that relative abundances of most of the metabolites in this class were lower in a treatment than those in another between the two treatments; a gray box denotes no significance between two treatments.

We also compared the metabolic differences between buds of the thermotolerant seeds (C106) and thermosensitive seeds (C62) that were germinated at $21^{\circ} \mathrm{C}$, which led to the selection of 2175 candidate metabolites of which 39 were putatively identified (Supplemental Table S2, Table S3, Table S5 and Figure S2B). C106 had higher concentrations of fatty acid derivatives (arachidic acid, stearic acid, 9-octadecenoic acid, (E)-, palmitic acid, 1-monolinolein, lignoceric acid) and meso-erythritol than C62 (Table 2 and Figure 3). However, C62 accumulated higher levels of some organic acids (caffeic acid, pyruvic acid, glyceric acid, quininic acid, butanedioic acid, L-threonic acid, 4-hydroxybutanoic acid, malic acid), amino acids (4-aminobutanoic acid, phenylalanine, L-isoleucine, L-5-oxoproline, L-threonine, L-alanine, 
L-glutamic acid, L-valine, L-aspartic acid, L-proline, L-ornithine), sugars (glucose and fructose), oleic acid, (Z)-, sterols (campesterol, stigmasterol), terpenoids ( $\alpha$-tocopherol, lactucopicrin) and polyphenols (dihydroxybenzoic acid hexoside, dihydroxybenzoic acid, caffeoylquinic acid, caffeoylquinic acid hexoside, dihydrocaffeic acid hexose, caffeoyl-hexose isomer 1) than C106 (Table 2 and Figure 3). Overall, the thermosensitive cultivar s13k0062 tended to accumulate more significant amounts of phenolic compounds, amino acids, organic acids, sugars, terpenoids and sterols during germination.

Table 2. Differential metabolites extracted from germinated buds of S15k0106 (C106) and S13k0062 (C62) seeds.

\begin{tabular}{|c|c|c|c|c|}
\hline No. & Compound & Log2 (FC C106/C62) & $p$-Value & VIP Value \\
\hline GC_89 & Pyruvic acid & -1.83 & $4.86 \times 10^{-5}$ & 1.12 \\
\hline GC_115 & L-Alanine & -2.13 & $5.94 \times 10^{-4}$ & 1.09 \\
\hline GC_185 & L-Valine & -2.34 & $4.27 \times 10^{-4}$ & 1.09 \\
\hline GC_200 & 4-Hydroxybutanoic acid & -3.46 & $8.85 \times 10^{-5}$ & 1.11 \\
\hline GC_233 & L-Isoleucine & -1.83 & $2.10 \times 10^{-5}$ & 1.13 \\
\hline GC_236 & L-Proline & -5.33 & $6.79 \times 10^{-5}$ & 1.12 \\
\hline GC_249 & Butanedioic acid & -2.69 & $5.53 \times 10^{-8}$ & 1.14 \\
\hline GC_258 & Glyceric acid & -1.85 & $2.17 \times 10^{-5}$ & 1.13 \\
\hline GC_290 & L-Threonine & -2.01 & $2.15 \times 10^{-8}$ & 1.14 \\
\hline GC_349 & Malic acid & -6.45 & $1.42 \times 10^{-9}$ & 1.14 \\
\hline GC_359 & meso-Erythritol & 1.56 & $9.35 \times 10^{-6}$ & 1.13 \\
\hline GC_362 & L-5-Oxoproline & -1.92 & $4.36 \times 10^{-7}$ & 1.14 \\
\hline GC_364 & L-Aspartic acid & -2.40 & $4.96 \times 10^{-7}$ & 1.14 \\
\hline GC_368 & 4-Aminobutanoic acid & -1.01 & $2.27 \times 10^{-4}$ & 1.10 \\
\hline GC_391 & L-Threonic acid & -2.19 & $7.27 \times 10^{-7}$ & 1.14 \\
\hline GC_425 & L-Ornithine & -5.37 & $1.53 \times 10^{-4}$ & 1.11 \\
\hline GC_427 & L-Glutamic acid & -2.28 & $1.70 \times 10^{-6}$ & 1.14 \\
\hline GC_430 & Phenylalanine & -1.27 & $4.23 \times 10^{-8}$ & 1.14 \\
\hline GC_554 & Quininic acid & -1.88 & $1.27 \times 10^{-6}$ & 1.14 \\
\hline GC_557 & Fructose & -2.91 & $6.58 \times 10^{-6}$ & 1.13 \\
\hline GC_569 & Glucose & -1.67 & $3.44 \times 10^{-5}$ & 1.12 \\
\hline GC_606 & Caffeic acid & -1.25 & $4.12 \times 10^{-6}$ & 1.13 \\
\hline GC_627 & Palmitic acid & 2.91 & $7.42 \times 10^{-8}$ & 1.14 \\
\hline GC_689 & 9-Octadecenoic acid, (E)- & 3.21 & $5.83 \times 10^{-6}$ & 1.13 \\
\hline GC_692 & Oleic acid, $(Z)-$ & -44.26 & $5.41 \times 10^{-15}$ & 1.14 \\
\hline GC_702 & Stearic acid & 3.77 & $1.55 \times 10^{-8}$ & 1.14 \\
\hline GC_786 & Arachidic acid & 3.84 & $1.33 \times 10^{-9}$ & 1.14 \\
\hline GC_874 & 1-Monolinolein & 1.43 & $2.11 \times 10^{-7}$ & 1.14 \\
\hline GC_897 & Lignoceric acid & 1.17 & $1.18 \times 10^{-4}$ & 1.11 \\
\hline GC_948 & $\alpha$-Tocopherol & -1.56 & $1.19 \times 10^{-4}$ & 1.11 \\
\hline GC_965 & Campesterol & -1.23 & $8.03 \times 10^{-5}$ & 1.12 \\
\hline GC_968 & Stigmasterol & -1.35 & $6.34 \times 10^{-5}$ & 1.12 \\
\hline LC_443 & Caffeoylquinic acid & -2.65 & $2.06 \times 10^{-4}$ & 1.10 \\
\hline LC_1072 & Dihydrocaffeic acid hexose & -3.52 & $6.67 \times 10^{-10}$ & 1.14 \\
\hline LC_1096 & Dihydroxybenzoic acid & -2.25 & $1.85 \times 10^{-7}$ & 1.14 \\
\hline LC_1152 & Caffeoyl-hexose 1 & -3.97 & $7.57 \times 10^{-9}$ & 1.14 \\
\hline LC_1736 & Lactucopicrin & -2.28 & $8.49 \times 10^{-6}$ & 1.13 \\
\hline LC_1777 & Dihydroxybenzoic acid hexoside & -2.01 & $1.65 \times 10^{-7}$ & 1.14 \\
\hline LC_4473 & Caffeoylquinic acid hexoside & -3.15 & $9.40 \times 10^{-8}$ & 1.14 \\
\hline
\end{tabular}

Note: Fold change of different metabolites between C106 and C62 is reported as Log2(FC C106/C62).

\subsection{Metabolite Differences between Thermotolerant Seeds Germinated at High Temperature and Optimal Temperature}

For the comparison of C106 vs. HY106, a total number of 2445 of differential metabolites candidate, among them, 41 were putatively annotated (Supplemental Tables S2, S3 and S6, and Figure S2C). Compared to s15k0106 buds germinated at $21^{\circ} \mathrm{C}$ (C106), we observed higher accumulation of organic acids, including tricarboxylic acid cycle (TCA) intermediates (butanedioic acid, fumaric acid, malic acid, citric acid), pyruvic acid, 4-hydroxybutanoic acid, quininic acid, glycolic acid, lactic acid, tartaric acid, glyceric acid, caffeic acid, amino acids (e.g., 4-aminobutanoic acid, phenylalanine, glycine, 
L-isoleucine, L-5-oxoproline, L-threonine, L-alanine, L-glutamic acid, L-valine, L-aspartic acid, L-proline, L-ornithine, L-methionine, L-leucine), sesquiterpene lactones (lactucopicrin), carbohydrates, including fructose, xylitol, arabinose, 1-(sn-Glycero-3-phospho)-1D-myo-inositol and lipid derivatives (including campesterol, stigmasterol, lignoceric acid, 9,12-octadecadienoic acid (Z, Z)-, palmitic acid, stearic acid, arachidic acid and 2-linoleoylglycerol) in s15k0106 seeds germinated at $35^{\circ} \mathrm{C}$ (HY106). However, only some carbohydrates, including meso-erythritol and arabinitol, were observed to be lower in seeds germinated at $35^{\circ} \mathrm{C}$ (HY106) than buds of the same cultivar at germinated at $21^{\circ} \mathrm{C}$ (C106; Table 3 and Figure 4).

Table 3. Differential metabolites detected between buds of $\mathrm{S} 15 \mathrm{k} 0106$ seeds germinated at $35^{\circ} \mathrm{C}$ (HY106) and $21^{\circ} \mathrm{C}(\mathrm{C} 106)$.

\begin{tabular}{|c|c|c|c|c|}
\hline No. & Compound & Log2 (FC HY106/C106) & $p$-Value & VIP Value \\
\hline GC_89 & Pyruvic acid & 8.18 & $1.16 \times 10^{-7}$ & 1.13 \\
\hline GC_95 & Lactic acid & 5.11 & $5.76 \times 10^{-8}$ & 1.13 \\
\hline GC_103 & Glycolic acid & 5.10 & $3.68 \times 10^{-7}$ & 1.13 \\
\hline GC_115 & L-Alanine & 7.60 & $8.95 \times 10^{-7}$ & 1.12 \\
\hline GC_122 & Glycine & 6.06 & $9.84 \times 10^{-6}$ & 1.12 \\
\hline GC_185 & L-Valine & 7.74 & $8.89 \times 10^{-7}$ & 1.12 \\
\hline GC_200 & 4-Hydroxybutanoic acid & 9.13 & $2.47 \times 10^{-7}$ & 1.13 \\
\hline GC_221 & L-Leucine & 8.36 & $1.41 \times 10^{-6}$ & 1.12 \\
\hline GC_233 & L-Isoleucine & 6.80 & $8.26 \times 10^{-8}$ & 1.13 \\
\hline GC_236 & L-Proline & 8.13 & $5.74 \times 10^{-6}$ & 1.12 \\
\hline GC_249 & Butanedioic acid & 6.81 & $1.79 \times 10^{-8}$ & 1.13 \\
\hline GC_258 & Glyceric acid & 7.88 & $3.10 \times 10^{-8}$ & 1.13 \\
\hline GC_270 & Fumaric acid & 9.83 & $2.10 \times 10^{-8}$ & 1.13 \\
\hline GC_290 & L-Threonine & 7.59 & $1.23 \times 10^{-8}$ & 1.13 \\
\hline GC_349 & Malic acid & 12.77 & $9.02 \times 10^{-10}$ & 1.13 \\
\hline GC_359 & meso-Erythritol & -2.49 & $5.77 \times 10^{-6}$ & 1.12 \\
\hline GC_361 & L-Methionine & 6.21 & $6.76 \times 10^{-8}$ & 1.13 \\
\hline GC_362 & L-5-Oxoproline & 7.61 & $3.01 \times 10^{-8}$ & 1.13 \\
\hline GC_364 & L-Aspartic acid & 8.73 & $2.22 \times 10^{-8}$ & 1.13 \\
\hline GC_368 & 4-Aminobutanoic acid & 6.54 & $5.38 \times 10^{-6}$ & 1.12 \\
\hline GC_425 & L-Ornithine & 11.77 & $2.35 \times 10^{-6}$ & 1.12 \\
\hline GC_427 & L-Glutamic acid & 8.47 & $5.08 \times 10^{-8}$ & 1.13 \\
\hline GC_430 & Phenylalanine & 6.41 & $2.81 \times 10^{-8}$ & 1.13 \\
\hline GC_439 & Tartaric acid & 8.50 & $2.50 \times 10^{-8}$ & 1.13 \\
\hline GC_457 & Arabinose & 4.81 & $3.56 \times 10^{-6}$ & 1.12 \\
\hline GC_468 & Xylitol & 4.18 & $2.86 \times 10^{-7}$ & 1.13 \\
\hline GC_484 & Arabinitol & -2.81 & $5.47 \times 10^{-6}$ & 1.12 \\
\hline GC_538 & Citric acid & 6.14 & $1.22 \times 10^{-7}$ & 1.13 \\
\hline GC_554 & Quininic acid & 4.25 & $4.31 \times 10^{-7}$ & 1.13 \\
\hline GC_557 & Fructose & 8.55 & $1.27 \times 10^{-6}$ & 1.12 \\
\hline GC_606 & Caffeic acid & 3.72 & $6.53 \times 10^{-4}$ & 1.07 \\
\hline GC_627 & Palmitic acid & 1.81 & $4.62 \times 10^{-5}$ & 1.10 \\
\hline GC_686 & 9,12-Octadecadienoic acid (Z, Z)- & 4.60 & $3.84 \times 10^{-7}$ & 1.13 \\
\hline GC_702 & Stearic acid & 1.56 & $7.71 \times 10^{-5}$ & 1.10 \\
\hline GC_786 & Arachidic acid & 1.60 & $1.01 \times 10^{-4}$ & 1.10 \\
\hline GC_863 & 2-Linoleoylglycerol & 4.88 & $7.03 \times 10^{-7}$ & 1.12 \\
\hline GC_897 & Lignoceric acid & 4.64 & $3.60 \times 10^{-7}$ & 1.13 \\
\hline GC_965 & Campesterol & 6.54 & $1.48 \times 10^{-7}$ & 1.13 \\
\hline GC_968 & Stigmasterol & 6.98 & $1.29 \times 10^{-7}$ & 1.13 \\
\hline LC_1736 & Lactucopicrin & 5.17 & $5.46 \times 10^{-7}$ & 1.12 \\
\hline LC_4802 & 1-(sn-Glycero-3-phospho)-1D-myo-inositol & 6.97 & $2.13 \times 10^{-4}$ & 1.09 \\
\hline
\end{tabular}

Note: Fold change of different metabolites between HY106 and C106 is reported as Log2(FC HY106/C106). 


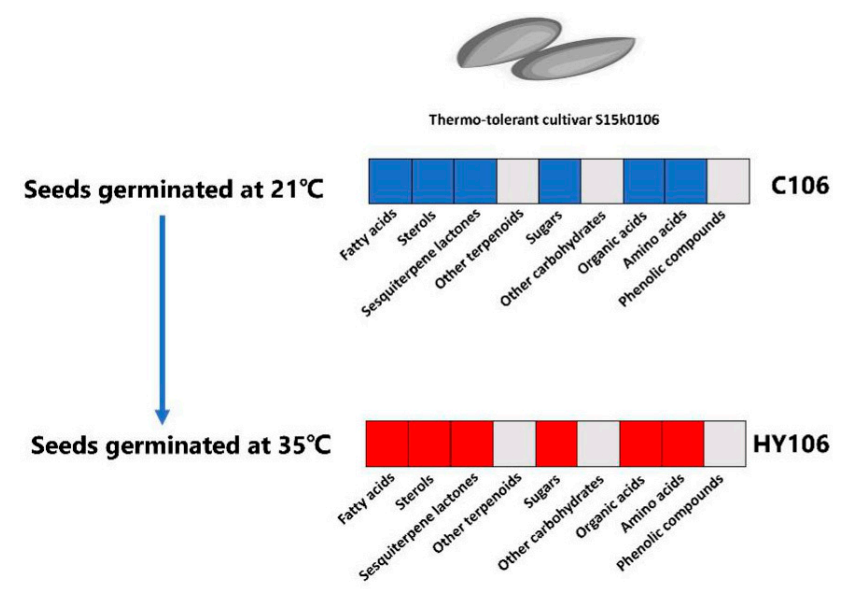

Figure 4. Differential metabolites detected between the thermotolerant lettuce seeds germinated at $21^{\circ} \mathrm{C}$ and $35^{\circ} \mathrm{C}$. Note: The heatmap indicates the differential metabolites detected between buds of S15k0106 seeds germinated at $21^{\circ} \mathrm{C}(\mathrm{C} 106)$ and $35{ }^{\circ} \mathrm{C}(\mathrm{HY} 106)$. A red box means that most of the metabolites in this class had higher relative abundances in HY106 compared to those in C106, while a blue box represents that relative abundances of most of the metabolites in this class were lower in C106 than those in HY106; a gray box denotes no significance between C106 and HY106.

\subsection{Metabolite Differences between Non-Germinated Seeds and Germinated Buds of Thermotolerant Cultivar at High Temperature}

We assessed the metabolic differences between non-germinated seeds and germinated buds of the thermotolerant cultivar after incubation at $35^{\circ} \mathrm{C}$ for $40 \mathrm{~h}$ (H106 vs. HY106). A total of 1220 differential metabolites candidates were detected, of which 39 candidates were putatively annotated (Supplemental Tables S2, S3 and S7 and Figure S2D). The non-germinated seeds had higher concentrations of uracil, fatty acids (arachidic acid, stearic acid, palmitic acid, 1-monolinolein) and R-3-hydroxybutyric acid than the germinated seeds. However, we detected higher concentrations of some organic acids (including butanedioic acid, quininic acid, glycolic acid, pyruvic acid, citric acid, ribonic acid, L-threonic acid, glyceric acid, fumaric acid, tartaric acid, 4-hydroxybutanoic acid, malic acid, propanedioic acid), alcohols (glycerol, 1-hexacosanol), carbohydrates (fructose, arabinitol, meso-erythritol, xylitol), amino acids (L-methionine, glycine, L-ornithine, L-alanine, phenylalanine, L-valine, L-aspartic acid, L-isoleucine, L-threonine, L-glutamic acid, L-proline, L-5-oxoproline), dihydrocaffeic acid hexose and 15-deoxylactucin-8-sulfate in germinated buds than the non-germinated seeds (Table 4 and Figure 5).

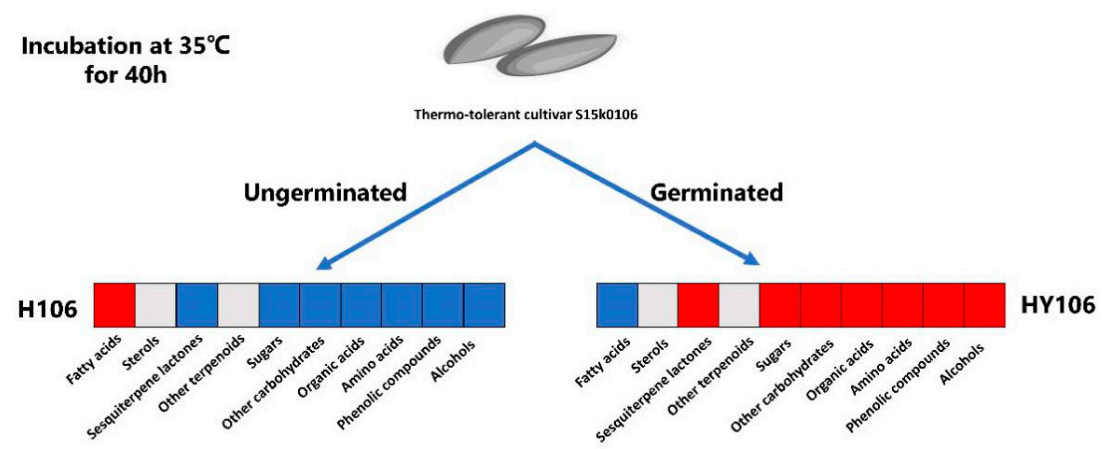

Figure 5. Differential metabolites detected between germinated buds and unterminated seeds of the thermal-tolerant lettuce cultivar. The heatmap indicates the differential metabolites between buds of S15k0106 germinated at $35{ }^{\circ} \mathrm{C}$ (HY106) and seeds of S15k0106 that did not germinate at $35{ }^{\circ} \mathrm{C}$ (H106). A red box means that most of the metabolites in this class had higher relative abundances in treatment compared to those in another, while a blue box represents that relative abundances of most of the metabolites in this class were lower in treatment than those in another; a gray box denotes no significance between H106 and HY106. 
Table 4. Differential metabolites between buds of S15k0106 germinated at $35^{\circ} \mathrm{C}$ (HY106) and seeds of S15k0106 that did not germinate at $35^{\circ} \mathrm{C}(\mathrm{H} 106)$.

\begin{tabular}{|c|c|c|c|c|}
\hline No. & Compound & Log2 (FC H106/HY106) & $p$-Value & VIP Value \\
\hline GC_103 & Glycolic acid & -2.29 & $2.12 \times 10^{-5}$ & 1.23 \\
\hline GC_108 & Pyruvic acid & -2.40 & $1.56 \times 10^{-5}$ & 1.23 \\
\hline GC_115 & L-Alanine & -3.14 & $2.64 \times 10^{-5}$ & 1.23 \\
\hline GC_122 & Glycine & -2.68 & $1.02 \times 10^{-4}$ & 1.22 \\
\hline GC_146 & R-3-Hydroxybutyric acid & 1.78 & $5.14 \times 10^{-3}$ & 1.12 \\
\hline GC_177 & Propanedioic acid & -34.26 & $5.88 \times 10^{-12}$ & 1.24 \\
\hline GC_185 & L-Valine & -3.42 & $2.20 \times 10^{-5}$ & 1.23 \\
\hline GC_200 & 4-Hydroxybutanoic acid & -5.27 & $4.09 \times 10^{-3}$ & 1.13 \\
\hline GC_223 & Glycerol & -1.07 & $3.67 \times 10^{-3}$ & 1.14 \\
\hline GC_233 & L-Isoleucine & -3.66 & $1.03 \times 10^{-5}$ & 1.23 \\
\hline GC_236 & L-Proline & -4.53 & $2.99 \times 10^{-7}$ & 1.24 \\
\hline GC_249 & Butanedioic acid & -1.14 & $4.45 \times 10^{-3}$ & 1.13 \\
\hline GC_258 & Glyceric acid & -3.90 & $1.87 \times 10^{-6}$ & 1.24 \\
\hline GC_261 & Uracil & 2.41 & $2.39 \times 10^{-3}$ & 1.15 \\
\hline GC_270 & Fumaric acid & -4.54 & $1.01 \times 10^{-5}$ & 1.23 \\
\hline GC_290 & L-Threonine & -3.72 & $5.43 \times 10^{-6}$ & 1.24 \\
\hline GC_349 & Malic acid & -6.99 & $1.50 \times 10^{-8}$ & 1.24 \\
\hline GC_359 & meso-Erythritol & -1.73 & $4.82 \times 10^{-4}$ & 1.19 \\
\hline GC_361 & L-Methionine & -2.00 & $6.25 \times 10^{-4}$ & 1.19 \\
\hline GC_362 & L-5-Oxoproline & -4.81 & $1.07 \times 10^{-7}$ & 1.24 \\
\hline GC_364 & L-Aspartic acid & -3.44 & $8.99 \times 10^{-6}$ & 1.23 \\
\hline GC_391 & L-Threonic acid & -3.20 & $1.49 \times 10^{-5}$ & 1.23 \\
\hline GC_425 & L-Ornithine & -2.72 & $2.15 \times 10^{-6}$ & 1.24 \\
\hline GC_427 & L-Glutamic acid & -3.80 & $3.32 \times 10^{-6}$ & 1.24 \\
\hline GC_430 & Phenylalanine & -3.35 & $7.81 \times 10^{-6}$ & 1.23 \\
\hline GC_439 & Tartaric acid & -5.25 & $6.12 \times 10^{-8}$ & 1.24 \\
\hline GC_468 & Xylitol & -2.46 & $9.96 \times 10^{-5}$ & 1.22 \\
\hline GC_484 & Arabinitol & -1.37 & $1.75 \times 10^{-4}$ & 1.21 \\
\hline GC_513 & Ribonic acid & -2.90 & $4.47 \times 10^{-5}$ & 1.22 \\
\hline GC_538 & Citric acid & -2.77 & $1.19 \times 10^{-4}$ & 1.22 \\
\hline GC_554 & Quininic acid & -1.95 & $3.91 \times 10^{-5}$ & 1.23 \\
\hline GC_557 & Fructose & -1.80 & $8.22 \times 10^{-3}$ & 1.10 \\
\hline GC_627 & Palmitic acid & 1.68 & $6.45 \times 10^{-5}$ & 1.22 \\
\hline GC_702 & Stearic acid & 1.94 & $3.32 \times 10^{-5}$ & 1.23 \\
\hline GC_786 & Arachidic acid & 2.00 & $2.69 \times 10^{-5}$ & 1.23 \\
\hline GC_874 & 1-Monolinolein & 1.54 & $1.22 \times 10^{-4}$ & 1.21 \\
\hline GC_917 & 1-Hexacosanol & -2.44 & $4.29 \times 10^{-6}$ & 1.24 \\
\hline LC_1072 & Dihydrocaffeic acid hexose & -1.69 & $7.35 \times 10^{-5}$ & 1.22 \\
\hline LC_1239 & 15-deoxylactucin-8-sulfate & -4.41 & $3.54 \times 10^{-7}$ & 1.24 \\
\hline
\end{tabular}

Note: Fold change of different metabolites between H106 and HY106 is reported as Log2(FC H106/HY106).

\section{Discussion}

\subsection{Germination of Lettuce Germplasms is Inhibited by High Temperature}

Lettuce seed germination is dramatically inhibited at high temperatures and will induce thermodormancy; the resulting poor seed germination and thermodormancy have detrimental effects on lettuce production in the field [17]. In this study, the average germination percentage of 304 lettuce accessions decreased significantly when the incubation temperature increased from $21^{\circ} \mathrm{C}$ to $35^{\circ} \mathrm{C}$. These results are in line with previous studies, which suggested that lettuce seed germination was hampered at temperatures between $25^{\circ} \mathrm{C}$ and $30^{\circ} \mathrm{C}$, depending on the genotype $[9,18,19]$. As the largest producer of lettuce worldwide, China contributes $56 \%$ of total world production [20]. In southern regions of China, the main area of lettuce production, over 30 days per year have a daily maximum 
temperature higher than $30^{\circ} \mathrm{C}$ [21]. Lettuce genotypes differ greatly in their ability to germinate at high temperatures [4]. Therefore, there is an urgent need to identify lettuce cultivars that exhibit thermotolerance. In this study, three lettuce accessions showed great seed germination performance under thermal stress. the highest relative percent germination among the commercial cultivars was observed in S15K0154 at both $28^{\circ} \mathrm{C}(90.61 \%)$ and $35^{\circ} \mathrm{C}(19.86 \%)$; the relative percent germination of S15K0123 was $107 \%$ at $28{ }^{\circ} \mathrm{C}$, implying that heat stress $\left(28^{\circ} \mathrm{C}\right)$ had no effect or rather induced germination; it is worth mentioning that the entry s15k0106 (W6 29844, now designated as PI 667844) is a cultivar named 'Florida Buttercrisp' [22], which is from a warm area and, as its name implies, had the highest germination rate at $35^{\circ} \mathrm{C}$. These results suggest that the three accessions have strong potential use in the breeding of heat-resistant lettuce. Among the accessions, we observed that non-commercial cultivars had a lower average relative percent germination than the commercial cultivars at both $28^{\circ} \mathrm{C}$ $(47.85 \%$ vs. $62.03 \%)$ and $35^{\circ} \mathrm{C}(1.13 \%$ vs. $1.58 \%)$. This result suggested that artificial selection plays an important role in improving germination rates in lettuce under heat stress.

Seed color had been previously reported to be associated with seed germination, dark-seeded lettuce is believed to have a higher percent of germination and increased seed vigor as well as lower pathogen susceptibility than white-seeded lettuce [23]. In this study, no relationship was observed between seed coats color and germination rate at different germination temperatures, suggesting that seed color is not a reliable predictor of final germination under heat stress conditions. The relationship between seed weight and vigor is controversial. In some crops (such as soybean and wheat), high seed weight has been linked to high seed vigor [24,25]. However, no relationship was observed in lettuce under cold conditions [26]. In this study, there was a negative relationship between relative percent germination and 100 -grain weight at $28^{\circ} \mathrm{C}\left(\mathrm{R}^{2}=-0.397, p<0.01\right)$, but not at $35^{\circ} \mathrm{C}$, suggesting that seed weight might be a useful predictor of germination at $28^{\circ} \mathrm{C}$. Further germination experiments at $21^{\circ} \mathrm{C}$ to $35^{\circ} \mathrm{C}$ are therefore required to explore higher correlation coefficients. There are several different opinions about the center of origin of cultivated lettuce. According to Lindqvist, it probably originated from Egypt [27]. According to Vavilov, cultivated lettuce originated in the Mediterranean area [28]. There was a single domestication event for cultivated lettuce that occurred $\sim 10,800$ years before present in the Kurdistan-Mesopotamia area [29,30]. In this study, the accessions from tropical countries, such as Vietnam, Kenya, and Laos, had a higher average relative percent germination at $28^{\circ} \mathrm{C}$ (Supplementary Table S1). However, the germination assessment of lettuce germplasms from the world at different temperatures showed no obvious relationship between the germination of lettuce seed and the original seed region.

\subsection{Metabolic Differences between Thermal-Sensitive and Thermal-Tolerant Cultivars during Germination at Proper Temperature}

Seed germination is a complex physiological and metabolic process that involves different classes of metabolites, including amino acids, organic acids, available sugars, antioxidants and lipids [31-33]. In this study, the total abundances of organic acids, amino acids and carbohydrates were higher in thermosensitive cultivar s13k0062 during germination, when compared to thermotolerant cultivar s15k0106. The reason could be that seed germination processes increase degradation of lipids to fatty acids, proteins to free amino acids and starch to available sugars, and markedly increase the accumulation of organic acids [34,35]. Moreover, we observed that the buds of 's13k0062' tended to accumulate more significant amounts of phenolic compounds and terpenoids compared to 'S15k0106' during germination at $21^{\circ} \mathrm{C}$. In the early stage of germination, the seed must activate the antioxidant enzyme system to accumulate antioxidants (such as phenolic compounds and $\alpha$-tocopherol) in order to scavenge excess reactive oxygen species and repair damage to the cell membrane [36]. The mevalonate pathway has been reported to be associated with lettuce seed germination. Isoprenoid biosynthesized via this pathway serves as a precursor for terpenoids (such as triterpenoids and sesquiterpenoids) and steroids [2]. These observations may explain the increased accumulation 
of terpenoids and phenolic compounds as antioxidants during germination in the seeds of the thermosensitive cultivar in this study.

\subsection{Metabolite Requirements for Thermo-Tolerant Seeds Germination under Thermal Stress}

Combined using GC/MS and UPLC-IMS-QTOF/MS platforms, we profiled the primary and secondary metabolites between the seed samples of thermotolerant lettuce cultivar of germinated at appropriate temperature $\left(21^{\circ} \mathrm{C}\right)$ and heat temperature $\left(35^{\circ} \mathrm{C}\right)$. We observed that thermotolerant lettuce cultivar accumulated higher concentrations of amino acids, organic acids, sugars, sesquiterpene lactones, sterols and fatty acids derivatives during the germination at $35^{\circ} \mathrm{C}$ compared to germinated at $21^{\circ} \mathrm{C}$. Amino acids and some carbohydrates are recognized as osmotic substances; the higher accumulation in seeds under thermal stress may contribute to the stabilization of membranes and cellular osmotic pressure [37]. In addition, increased the contents of amino acids (such as glycine, L-alanine, proline, L-5-oxoproline, L-glutamic acid, and L-aspartic acid and L-ornithine) and sugars (e.g., fructose, xylitol and arabinose) function as compatible solute properties in Arabidopsis in response to temperature stress [37]. Aromatic amino acid (phenylalanine) is an important precursor of the phenylpropanoid pathway, increased level of L-phenylalanine might be the reason for the accumulation of phenolic compounds downstream. 4-aminobutanoic acid plays a crucial role in reactive oxygen species scavenging by regulating the gene expression of $\mathrm{H}_{2} \mathrm{O}_{2}$-producing genes (NADPH oxidase, peroxidase and amine oxidase) in the plant under stress [38]. Branched-chain amino acids, including valine, leucine and isoleucine as well as other amino acids that share the biosynthetic pathway, such as threonine and methionine function as compatible osmolytes under stress condition [39]. Additionally, these amino acids play as alternative electron donors for the mitochondrial electron transport chain under stress conditions [40].

Our results showed that pyruvic acid and TCA intermediates, such as butanedioic acid, fumaric acid, malic acid and citric acid, had higher accumulation in HY106 compared to C106. Pyruvic acid plays an essential precursor for the TCA cycle, and its accumulation results in the increased levels of TCA intermediates. In addition, the previous study reported that the activation of 4-aminobutanoic acid shunt resulted in TCA intermediates accumulation in Arabidopsis in response to dehydration due to the interplay between the 4-aminobutanoic acid shunt and the TCA cycle through various bypasses [41].

Notably, we observed that N106 seeds contain higher concentrations of the unsaturated long-chain fatty acids and saturated long-chain fatty acids than those in seeds of the thermosensitive cultivar. These results imply that heat-resistant of lettuce seed germination might be associated with lipids metabolism. Lipids are energy sources in seeds during embryo development, and the accumulation of high abundances of fatty acids is associated with energy metabolism in seeds [42]. Moreover, fatty acids and sterols are also involved in plant thermotolerant metabolism. Fatty acids are the major forms of the phospholipid bilayer, and their composition is crucial for maintaining membrane stability under stress [43]. Sterols act as regulators for the fluidity and permeability of cellular membranes [44]. In this study, we observed some steroids (stigmasterol and campesterol) accumulated at high levels in the seeds of the thermotolerant cultivar germinated at $35{ }^{\circ} \mathrm{C}$ (HY106). These findings are in line with previous observation in a perennial grass, hard fescue (Festuca Trachyphylla): ethyl sterols (fucosterol, stigmasterol, sitosterol, avenasterol) are positively associated with heat tolerance [45]. Fatty acid length in the stable membrane governs the thickness of lipid bilayers and tends to match the thickness of the membrane proteins [46]. To maintain membrane stability, s15k0106 seeds germinated at $35^{\circ} \mathrm{C}$ (HY106) accumulated high concentration of long-chain fatty acid, such as lignoceric acid (C24), arachidic acid (C20), stearic acid (C18) and palmitic acid (C16). These results suggested that seeds of thermotolerant cultivar s15k0106 might employ different lipid metabolic strategies under thermal stress, including increased accumulation of long-chain fatty acids and sterols (stigmasterol and campesterol). These metabolic strategies could be beneficial for maintaining a proper lipid environment 
in the seed membrane for maintaining membrane stability and functionality during seeds adaptation to thermal stress [47].

Interestingly, we observed significantly higher accumulation of organic acids, amino acids, sesquiterpene lactones, lipids and carbohydrates in the buds of the thermotolerant accession s15k0106 when germinated at $35^{\circ} \mathrm{C}$ (HY106) than when germinated at $21^{\circ} \mathrm{C}(\mathrm{C} 106)$. A similar metabolic strategy was detected between s13k0062 buds germinated at $21^{\circ} \mathrm{C}(\mathrm{C} 62)$ and s15k0106 buds germinated at $21^{\circ} \mathrm{C}$ (C106). Twenty-three differential metabolites were common to both of these comparisons (HY106 vs. $\mathrm{C} 106$ and $\mathrm{C} 106$ vs. $\mathrm{C} 62)$, suggesting that the thermotolerant cultivar s15k0106 may employ a similar metabolic strategy when germinated at high temperatures $\left(35^{\circ} \mathrm{C}\right)$ as the thermosensitive cultivar s13k0062 when germinated at $21^{\circ} \mathrm{C}$. While the thermosensitive s13k0062 seeds accumulated high levels of antioxidants (i.e., phenolic acids and $\alpha$-tocopherol) during germination, and the accumulation of phenolic compounds were observed to be lower in thermotolerant s15k0106 seeds during germination at $35^{\circ} \mathrm{C}$ compared to $21^{\circ} \mathrm{C}$. The reason could be that the thermotolerance of lettuce seeds undergoing germination is associated with the levels of reactive oxygen species in the seed. The levels of activated oxygen species were higher in the thermosensitive cultivar than the thermotolerant cultivar, which would lead to poorer heat resistance in the thermosensitive cultivar. Collectively, thermosensitive and thermotolerant cultivars employ distinctive metabolic strategies in response to heat stress. This work identified a large number of potential metabolic targets for further in-depth investigation of acquired seed tolerance to temperature stress. Moreover, these differential metabolites could be potential biomarkers for further screening of thermotolerant lettuce varieties for breeding projects.

\section{Materials and Methods}

\subsection{Evaluate Seed Germination under Different Temperature}

Used in the current study were 304 lettuce accessions, of which 257 were kindly offered by the Western Region Plant Introduction Station of the US Department of Agriculture, Pullman, WA, USA; 17 were provided by World Vegetable Centre, Taiwan, China; 30 commercial cultivars were purchased from the local market in Shanghai, China. Seeds were stored at $-20^{\circ} \mathrm{C}$ until starting the germination test.

For the germination test, three replicates of 100 seeds per accession or cultivar were placed in petri dishes $(120 \times 120 \mathrm{~mm})$ on a single layer of Whatman \#1 filter paper. A thin sponge was placed under the filter paper to retain moisture, and $4.5 \mathrm{~mL}$ of deionized water was added. The petri dishes were covered with lids to prevent evaporation then placed in incubators at $21^{\circ} \mathrm{C}$ (proper temperature for lettuce seeds germination), $28^{\circ} \mathrm{C}$ (upper limit temperature for lettuce seed) or $35^{\circ} \mathrm{C}$ (upper limit temperature for thermotolerant cultivar seed) for $40 \mathrm{~h}$ in the dark to mimic the conditions of the soil at germination. Seed germination was recorded as the emergence of a radicle after incubation. Analysis of variance was performed on the germination data.

\subsection{Two Accessions Are Selected for Metabolic Profiling}

Two accessions, 's13k0062' and 's15k0106', were selected for metabolic profiling based on their germination performance under different temperatures. At $21^{\circ} \mathrm{C}$, both 's13k0062' and 's15k0106' germinated well with the germination percentages of $92.67 \%$ and $76.0 \%$, respectively. However, at $28{ }^{\circ} \mathrm{C}$, only less than $2 \%$ of the seeds of 's $13 \mathrm{k} 0062^{\prime}$ ' germinated, while the germination percentage of 's $15 \mathrm{k} 0106^{\prime}$ remained at $74.67 \%$, almost the same as that at $21^{\circ} \mathrm{C}$. Still, at $35{ }^{\circ} \mathrm{C}$, 's $13 \mathrm{k} 0062^{\prime}$ had $0 \%$ germination, while 's15k0106' had 39.67\% germination, the highest among all 304 accessions evaluated (Supplemental Table S1). Thus, we can say 's13k0062' is thermosensitive and 's15k0106' thermotolerant.

Four replicates of 100 seeds per cultivar were placed in an incubator to mimic seed germination using the method described above at $21^{\circ} \mathrm{C}$ and $35^{\circ} \mathrm{C}$ for $40 \mathrm{~h}$. After incubation, all samples were collected and stored at $-80^{\circ} \mathrm{C}$ for metabolite extraction. 


\subsection{Metabolite Profiling}

\subsubsection{GC-MS Analysis}

A recently published extraction protocol [15] was followed for preparing samples for GC-MS analysis. Briefly, lettuce leaf sample $(200 \mathrm{mg})$ was weighed and ground into fine powders in liquid nitrogen. Next, $1 \mathrm{~mL}$ ice-cold methanol: chloroform solution (3:1, v:v) was added. Samples were then vortexed at $6000 \mathrm{rpm}$ for $15 \mathrm{~s}$ (repeated three times), and an internal standard ( $20 \mu \mathrm{L}$ of $0.3 \mathrm{mg} \cdot \mathrm{mL}^{-1}$ L-2-chlorophenylalanine) was added. After that, samples were centrifuged at $15,000 \times g$ for $10 \mathrm{~min}$, and $300 \mu \mathrm{L}$ supernatant of each sample was transferred to a new vial and vacuum-dried at room temperature. For derivatization, $80 \mu \mathrm{L}$ of $20 \mathrm{mg} \cdot \mathrm{mL}^{-1}$ methoxyamine hydrochloride (dissolved in pyridine) was added to the vial, and samples were then transferred into an oven and incubated at $37^{\circ} \mathrm{C}$ for $1.5 \mathrm{~h}$. Then, $80 \mu \mathrm{L}$ of $\mathrm{N}, \mathrm{O}-\mathrm{Bis}($ trimethylsilyl)trifluoroacetamide solution with $1 \%$ trimethylchlorosilane was added and followed by incubation at $70^{\circ} \mathrm{C}$ for $1 \mathrm{~h}$. Supernatants of all samples were mixed, vacuum-dried and derivatized in the same manner to prepare QC samples. $1 \mu \mathrm{L}$ derived extract of each sample was analyzed by using an Agilent 7890A-5975C GC/MS system (Agilent J\&W Scientific, Folsom, CA, USA) coupled with a DB-5MS capillary column $(30 \mathrm{~m} \times 0.25 \mathrm{~mm} \times 0.25 \mu \mathrm{m})$. The GC conditions were set as inlet temperature, $280^{\circ} \mathrm{C}$; After $6.5 \mathrm{~min}$ of solvent delay, the initial GC oven temperature was $60^{\circ} \mathrm{C}$; $1 \mathrm{~min}$ after injection, the GC oven temperature was raised to $300{ }^{\circ} \mathrm{C}$ at $5{ }^{\circ} \mathrm{C} \mathrm{min}-1$, and maintained at $300{ }^{\circ} \mathrm{C}$ for $11 \mathrm{~min}$; transfer line temperature, $280{ }^{\circ} \mathrm{C}$; ion source temperature, $230{ }^{\circ} \mathrm{C}$; carrier gas $(\mathrm{He})$

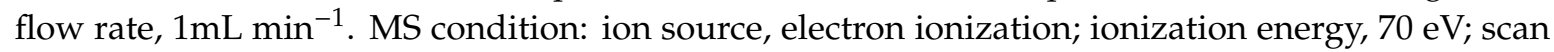
mode, full scan; scan range, $m / z$ 33-600. The mixture of Supelco C7-C40 saturated alkanes standard ran prior to the samples to calculate the retention index of each feature. The QC samples were run at the start, middle, and end of the analysis.

\subsubsection{UPLC-IMS-QTOF/MS Analysis}

The sample for UPLC-MS analysis was prepared and analyzed, as described previously [16]. In brief, lettuce seed samples were ground in liquid nitrogen into powder and then extracted with $1 \mathrm{~mL}$ methanol/water solution $(80: 20, v / v)$. Samples were then sonicated at $25^{\circ} \mathrm{C}$ for $30 \mathrm{~min}$ and kept in the refrigerator $\left(4^{\circ} \mathrm{C}\right)$ for $12 \mathrm{~h}$, followed by centrifugation at $12000 \times g$ for $10 \mathrm{~min} .500 \mu \mathrm{L}$ of the supernatant was collected for further analysis. QC samples were prepared by mixing the supernatant of all samples.

Samples $(3 \mu \mathrm{L})$ were then analyzed by an Acquity class UPLC coupled with Vion IMS QTOF MS (Waters, Corp. Milford, MA, USA) using an Acquity UPLC HSS T3 column $(100 \mathrm{~mm} \times 2.1 \mathrm{~mm}$, i, d.: $1.7 \mu \mathrm{m})$. Mobile phase: Water containing $0.1 \%$ formic acid (A) and acetonitrile containing $0.1 \%$ formic acid (B); flow rate: $0.4 \mathrm{~mL} \mathrm{~min}^{-1}$; UPLC conditions: gradient elution, $0-3 \mathrm{~min}, 0 \% \mathrm{~B} ; 3-3.1 \mathrm{~min}, 0-5 \% \mathrm{~B}$; 3.1-6 min, 5 to $20 \% \mathrm{~B}$; 6-11 $\mathrm{min}, 20-50 \% \mathrm{~B} ; 11-15 \mathrm{~min} 50$ to $100 \% \mathrm{~B}, 15-17 \mathrm{~min}, 100 \% \mathrm{~B}$, then initial conditions were restored for $5 \mathrm{~min}$ to equilibrate the column. For MS conditions, ion mode, negative; capillary voltage, $1.5 \mathrm{kV}$; source temperature, $115^{\circ} \mathrm{C}$; desolvation temperature, $500{ }^{\circ} \mathrm{C}$; desolvation gas flow, $1000 \mathrm{~L} \mathrm{~h}^{-1}$; scan range, $\mathrm{m} / \mathrm{z}$ 50-1000; collision energy, $20-40 \mathrm{eV}$. Data acquisition mode was performed in $\mathrm{HDMS}^{\mathrm{E}}$ (data-independent acquisition type in ion-mobility). A $5 \mu \mathrm{L}$ of $0.2 \mathrm{mg} \mathrm{mL}^{-1}$ EGCG was added as an internal standard. QC samples were inserted into each sample branch.

\subsection{Data Pre-Processing and Normalization}

Raw data acquired from GC/MS and UPLC-IMS-QTOF/MS were pre-processed by LECO Chroma TOF and Waters Progenesis QI, respectively. The raw data was performed in the pre-process procedures, including peak picking, baselining and alignment. Then, the pre-processing data matrix containing feature name (named as retention time and $\mathrm{m} / \mathrm{z}$ ), sample information (four biological replicates per sample), relative abundance (calculated by peak area) was prepared and submitted to MetaboAnalyst (https://www.metaboanalyst.ca/). After that, the data matrix was performed three categories of 
normalization, including normalization by median, log transformation and auto-scaling via online data analysis software MetaboAnalyst embedded algorithm.

\subsection{Data Analysis}

\subsubsection{Study Design}

Four comparisons between different groups were set to investigate the difference in metabolomes between the two accessions of 1 . dry seeds (N106 vs. N62, denoting 's15k0106' of and 's13k0062', respectively); 2 . the germinated seeds of the two accessions at $21^{\circ} \mathrm{C}(\mathrm{C} 106$ vs. $\mathrm{C} 62) ; 3$. the germinated seeds of 's15k0106' at $21^{\circ} \mathrm{C}$ and $35^{\circ} \mathrm{C}$ (HY106 vs. C106); and 4) the seeds of 's15k0106' germinated and non-germinated at $35^{\circ} \mathrm{C}$ (HY106 vs. H106). The study design is shown in Figure 6 .

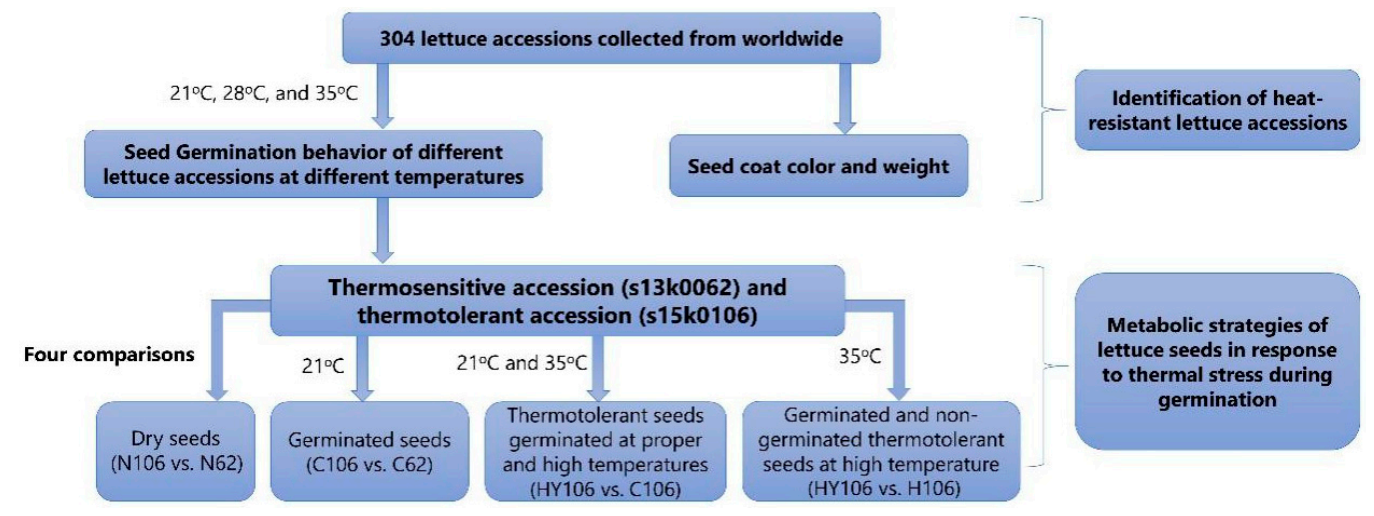

Figure 6. The workflow of the current study.

\subsubsection{Univariate and Multivariate Statistical Analysis}

The principal component analysis (PCA) of all samples and QCs was performed in SIMCA-P software (version 14.1, Sartorius Stedim Biotech, Umeå, Sweden). To identify potential variables that contribute to differentiation of four pairs of comparison, fold change and student's t-test value were calculated, and VIP value was calculated by conducting the partial least squares-discriminant analysis (PLS-DA). All these parameters were obtained by the embedded algorithm in MetaboAnalyst.

\subsubsection{Differential Metabolites Screening}

The differential metabolites were screened by parameters including fold change $>2$, VIP value $>1$, and $p$-value $<0.01$. Then, these 'candidates' were marked for compound identification to explore their biological roles during seed germination.

\subsubsection{Compound Identification}

Candidates from GC-MS were identified through compared with reference and online database including our in-house lettuce metabolites library [15], NIST 2014 (Similarity > 800) and Golm Metabolome Database (CV of Retention Index $<1.5 \%$ ); candidates from UPLC-IMS-QTOF/MS metabolites were identified through compared with our in-house database [16], which included mass to charge, retention time, $\mathrm{MS}^{2}$ fragments. All matching results were then supervised manually.

\section{Conclusions}

In conclusion, the germination of lettuce germplasm reduces as temperature increases. Artificial selection has improved the germination rates of lettuce under heat stress. Our comparative metabolomic analysis revealed that the accumulation of organic acids, amino acids, terpenoids, phenolic compounds, carbohydrates and lipids are involved in lettuce seed germination and thermoinhibition. Thermosensitive seeds accumulated more significant amounts of organic acids, amino acids, sugars, 
sterols, phenolic compounds and terpenoids compared to thermotolerant seeds at $21^{\circ} \mathrm{C}$. Thermotolerant lettuce cultivar accumulated higher concentrations of amino acids, organic acids, sugars, sesquiterpene lactones, sterols and fatty acids derivatives during the germination at $35^{\circ} \mathrm{C}$ compared to germinated at $21{ }^{\circ} \mathrm{C}$. Overall, our findings indicate the complexity of lettuce germination under abiotic stress conditions. Seeds of thermal-sensitive and thermal-tolerant cultivars employed different metabolic strategies in response to thermal stress during germination. Moreover, heat-resistant accessions were identified for future breeding programs that aim to develop new cultivars suitable for extended summer production in temperate or tropical climates when soil temperatures are higher than the optimal germination temperature. Further studies are now required to assess the ability of these accessions to germinate rapidly at higher temperatures under field conditions.

Supplementary Materials: Supplementary materials can be found at http://www.mdpi.com/1422-0067/21/4/1481/s1.

Author Contributions: Conceptualization, S.W.; methodology, S.W. and X.Y.; software, X.Y.; formal analysis, S.W., X.Y., G.H., G.G. and H.L.; resources, S.W. and X.Y; writing-original draft preparation, S.W. and X.Y.; writing-review and editing, L.L. and J.H.; visualization, X.Y.; supervision, D.H. and P.L. All authors have read and agreed to the published version of the manuscript.

Funding: This research was funded by the Shanghai Science and Technology Support Project (Grant No. 19391900600), the Seed Industry Development Project of Shanghai, China (Grant No. 2016, 1-8), Sharing Platform for National Crop Germplasm Resources (Shanghai; Grant No. NICGR2019-21), the Shared Platform of Crop Germplasm Resources in Shanghai (Grant No. 18DZ2293700), the Central Public-Interest Scientific Institution Basal Research Fund (Grant No. Y2020XK01 and No. Y2018YJ25) and local financial funding from the National Agriculture Science and Technology Center, Chengdu (Grant No. NASC2019AR01).

Acknowledgments: We acknowledge the Germplasm Resources Information Network (USDA) for kindly providing the lettuce accessions used in this study.

Conflicts of Interest: The authors declare no conflict of interest. The funders had no role in the design of the study; in the collection, analyses or interpretation of data; in the writing of the manuscript, or in the decision to publish the results.

\section{Abbreviations}

Quality control

Mass spectrometry

QC

Shanghai Agricultural Gene Center

MS

Shanghai Agricultural Gene Center

SAGC

Gas chromatography-mass spectrometry

GC/MS

Ultra-performance liquid chromatography-ion

UPLC-IMS-QTOF/MS

mobility spectrometry quadrupole time-of-flight

mass spectrometry

Principal component analysis

PCA

Partial least squares-discriminant analysis $\quad$ PLS-DA

Tricarboxylic acid cycle $\quad$ TCA

Variable importance in projection VIP

\section{References}

1. Abeles, F.B.; Lonski, J. Stimulation of lettuce seed germination by ethylene. Plant Physiol. 1969, 44, $277-280$. [CrossRef]

2. Wang, W.Q.; Song, B.Y.; Deng, Z.J.; Wang, Y.; Liu, S.J.; Moller, I.M.; Song, S.Q. Proteomic analysis of lettuce seed germination and thermoinhibition by sampling of individual seeds at germination and removal of storage proteins by polyethylene glycol fractionation. Plant Physiol. 2015, 167, 1332-1350. [CrossRef]

3. Huo, H.; Dahal, P.; Kunusoth, K.; McCallum, C.M.; Bradford, K.J. Expression of 9-cis-epoxycarotenoid dioxygenase 4 is essential for thermoinhibition of lettuce seed germination but not for seed development or stress tolerance. Plant Cell 2013, 25, 884-900. [CrossRef]

4. Lafta, A.; Mou, B. Evaluation of lettuce genotypes for seed thermotolerance. Hortscience 2013, 48, 708-714. [CrossRef] 
5. Nascimento, W.M.; Cantliffe, D.J.; Huber, D.J. Thermotolerance in lettuce seeds: Association with ethylene and endo- $\beta$-mannanase. J. Am. Soc. Hortic. Sci. 2000, 125, 518-524. [CrossRef]

6. Contreras, S.; Bennett, M.A.; Metzger, J.D.; Tay, D.; Nerson, H. Red to far-red ratio during seed development affects lettuce seed germinability and longevity. HortScience 2009, 44, 130-134. [CrossRef]

7. Borthwick, H.A.; Hendricks, S.B.; Parker, M.W.; Toole, E.H.; Toole, V.K. A Reversible photoreaction controlling seed germination. Proc. Natl. Acad. Sci. USA 1952, 38, 662-666. [CrossRef]

8. Toyomasu, T.; Kawaide, H.; Mitsuhashi, W.; Inoue, Y.; Kamiya, Y. Phytochrome regulates gibberellin biosynthesis during germination of photoblastic lettuce seeds. Plant Physiol. 1998, 118, 1517-1523. [CrossRef]

9. Argyris, J.; Dahal, P.; Hayashi, E.; Still, D.W.; Bradford, K.J. Genetic variation for lettuce seed thermoinhibition is associated with temperature-sensitive expression of abscisic acid, gibberellin, and ethylene biosynthesis, metabolism, and response genes. Plant Physiol. 2008, 148, 926-947. [CrossRef] [PubMed]

10. Yoong, F.Y.; O’Brien, L.K.; Truco, M.J.; Huo, H.; Sideman, R.; Hayes, R.; Michelmore, R.W.; Bradford, K.J. Genetic variation for thermotolerance in lettuce seed germination is associated with temperature-sensitive regulation of ethylene response factor1 (ERF1). Plant Physiol. 2016, 170, 472-488. [CrossRef]

11. De Vos, R.C.; Moco, S.; Lommen, A.; Keurentjes, J.J.; Bino, R.J.; Hall, R.D. Untargeted large-scale plant metabolomics using liquid chromatography coupled to mass spectrometry. Nat. Protoc. 2007, 2, 778-791. [CrossRef]

12. Fiehn, O. Metabolomics - The link between genotypes and phenotypes. Plant Mol. Biol. 2002, 48, $155-171$. [CrossRef]

13. Garcia, C.J.; Garcia-Villalba, R.; Gil, M.I.; Tomas-Barberan, F.A. LC-MS untargeted metabolomics to explain the signal metabolites inducing browning in fresh-cut lettuce. J Agric. Food Chem. 2017, 65, 4526-4535. [CrossRef]

14. García, C.J.; Gil, M.I.; Tomas-Barberan, F.A. LC-MS untargeted metabolomics reveals early biomarkers to predict browning of fresh-cut lettuce. Postharvest Biol. Technol. 2018, 146, 9-17. [CrossRef]

15. Yang, X.; Feng, L.; Zhao, L.; Liu, X.; Hassani, D.; Huang, D. Effect of glycine nitrogen on lettuce growth under soilless culture: A metabolomics approach to identify the main changes occurred in plant primary and secondary metabolism. J. Sci. Food Agric. 2018, 98, 467-477. [CrossRef]

16. Yang, X.; Wei, S.; Liu, B.; Guo, D.; Zheng, B.; Feng, L.; Liu, Y.; Tomas-Barberan, F.A.; Luo, L.; Huang, D. A novel integrated non-targeted metabolomic analysis reveals significant metabolite variations between different lettuce (Lactuca sativa, L.) varieties. Hortic. Res. 2018, 5, 33. [CrossRef]

17. Gonai, T.; Kawahara, S.; Tougou, M.; Satoh, S.; Hashiba, T.; Hirai, N.; Kawaide, H.; Kamiya, Y.; Yoshioka, T. Abscisic acid in the thermoinhibition of lettuce seed germination and enhancement of its catabolism by gibberellin. J. Exp. Bot. 2004, 55, 111-118. [CrossRef]

18. Gray, D. Effects of temperature on the germination and emergence of lettuce (Lactuca sativa, L.) varieties. J. Hortic. Sci. 1975, 50, 349-361. [CrossRef]

19. Kozarewa, I.; Cantliffe, D.J.; Nagata, R.T.; Stoffella, P.J. High maturation temperature of lettuce seeds during development increased ethylene production and germination at elevated temperatures. J. Am. Soc. Hortic. Sci. 2006, 131, 564-570. [CrossRef]

20. FAO, U.N. Lettuce and Chicory Production of 2017. Available online: http://www.fao.org/faostat/en/\#data/ QC/visualize (accessed on 1 June 2019).

21. China Meteorological Administration. China Climate Bulletin 2018. Available online: http://www.cma.gov. cn/root7/auto13139/201903/t20190319_517664.html (accessed on 1 June 2019).

22. NPGS, U.A. Grin Gloval Database. Available online: https://npgsweb.ars-grin.gov/gringlobal/accessiondetail. aspx?id=1718215 (accessed on 14 October 2019).

23. Penaloza, P.; Ramirez-Rosales, G.; McDonald, M.B.; Bennett, M.A. Lettuce (Lactuca sativa, L.) seed quality evaluation using seed physical attributes, saturated salt accelerated aging and the seed vigour imaging system. Electron. J. Biotechnol. 2005, 8. [CrossRef]

24. Hoy, D.J.; Gamble, E.E. The effects of seed size and seed density on germination and vigor in soybean (Glycine max (L.) Merr.). Can. J. Plant Sci. 1985, 65, 1-8. [CrossRef]

25. Lafond, G.; Baker, R. Effects of genotype and seed size on speed of emergence and seedling vigor in nine spring wheat cultivars 1. Crop Sci. 1986, 26, 341-346. [CrossRef]

26. Grahn, C.M.; Hellier, B.C.; Benedict, C.; Miles, C.A. Screening USDA lettuce (Lactuca sativa, L.) germplasm for ability to germinate under cold conditions. Hortscience 2015, 50, 1155-1159. [CrossRef] 
27. Lindqvist, K. On the origin of cultivated lettuce. Hereditas 1960, 46, 319-350. [CrossRef]

28. De Vries, I.M. Origin and domestication of Lactuca sativa L. Genet. Resour. Crop Evol. 1997, 44, $165-174$. [CrossRef]

29. Meyer, R.S.; Purugganan, M.D. Evolution of crop species: Genetics of domestication and diversification. Nat. Rev. Genet. 2013, 14, 840-852. [CrossRef]

30. Zhang, L.; Su, W.Q.; Tao, R.; Zhang, W.Y.; Chen, J.J.; Wu, P.Y.; Yan, C.H.; Jia, Y.; Larkin, R.M.; Lavelle, D.; et al. RNA sequencing provides insights into the evolution of lettuce and the regulation of flavonoid biosynthesis. Nat. Commun. 2017, 8, 12. [CrossRef]

31. Fernandez-Orozco, R.; Piskula, M.K.; Zielinski, H.; Kozlowska, H.; Frias, J.; Vidal-Valverde, C. Germination as a process to improve the antioxidant capacity of Lupinus angustifolius L. var. Zapaton. Eur. Food Res. Technol. 2006, 223, 495-502. [CrossRef]

32. Dueñas, M.; Hernández, T.; Estrella, I.; Fernández, D. Germination as a process to increase the polyphenol content and antioxidant activity of lupin seeds (Lupinus angustifolius, L.). Food Chem. 2009, 117, 599-607. [CrossRef]

33. Guo, X.; Li, T.; Tang, K.; Liu, R.H. Effect of germination on phytochemical profiles and antioxidant activity of mung bean sprouts (Vigna radiata). J. Agric. Food Chem. 2012, 60, 11050-11055. [CrossRef]

34. Bewley, J.D. Seed germination and dormancy. Plant Cell 1997, 9, 1055. [CrossRef]

35. Sripriya, G.; Antony, U.; Chandra, T.S. Changes in carbohydrate, free amino acids, organic acids, phytate and $\mathrm{HCl}$ extractability of minerals during germination and fermentation of finger millet (Eleusine coracana). Food Chem. 1997, 58, 345-350. [CrossRef]

36. Wang, W.B.; Kim, Y.H.; Lee, H.S.; Kim, K.Y.; Deng, X.P.; Kwak, S.S. Analysis of antioxidant enzyme activity during germination of alfalfa under salt and drought stresses. Plant Physiol. Biochem. 2009, 47, 570-577. [CrossRef]

37. Kaplan, F.; Kopka, J.; Haskell, D.W.; Zhao, W.; Schiller, K.C.; Gatzke, N.; Sung, D.Y.; Guy, C.L. Exploring the temperature-stress metabolome of Arabidopsis. Plant Physiol. 2004, 136, 4159-4168. [CrossRef]

38. Shi, S.-Q.; Shi, Z.; Jiang, Z.-P.; Qi, L.-W.; Sun, X.-M.; Li, C.-X.; Liu, J.-F.; Xiao, W.-F.; Zhang, S.-G. Effects of exogenous GABA on gene expression of Caragana intermedia roots under $\mathrm{NaCl}$ stress: Regulatory roles for $\mathrm{H}_{2} \mathrm{O}_{2}$ and ethylene production. Plant Cell Environ. 2010, 33, 149-162. [CrossRef]

39. Joshi, V.; Joung, J.-G.; Fei, Z.; Jander, G. Interdependence of threonine, methionine and isoleucine metabolism in plants: Accumulation and transcriptional regulation under abiotic stress. Amino Acids 2010, 39, $933-947$. [CrossRef]

40. Araujo, W.L.; Tohge, T.; Ishizaki, K.; Leaver, C.J.; Fernie, A.R. Protein degradation-An alternative respiratory substrate for stressed plants. Trends Plant Sci. 2011, 16, 489-498. [CrossRef]

41. Urano, K.; Maruyama, K.; Ogata, Y.; Morishita, Y.; Takeda, M.; Sakurai, N.; Suzuki, H.; Saito, K.; Shibata, D.; Kobayashi, M.; et al. Characterization of the ABA-regulated global responses to dehydration in Arabidopsis by metabolomics. Plant J. 2009, 57, 1065-1078. [CrossRef]

42. Pujar, A.; Jaiswal, P.; Kellogg, E.A.; Ilic, K.; Vincent, L.; Avraham, S.; Stevens, P.; Zapata, F.; Reiser, L.; Rhee, S.Y.; et al. Whole-plant growth stage ontology for angiosperms and its application in plant biology. Plant Physiol. 2006, 142, 414-428. [CrossRef]

43. Gigon, A.; Matos, A.R.; Laffray, D.; Zuily-Fodil, Y.; Pham-Thi, A.T. Effect of drought stress on lipid metabolism in the leaves of Arabidopsis thaliana (ecotype Columbia). Ann. Bot. 2004, 94, 345-351. [CrossRef]

44. Schaller, H. The role of sterols in plant growth and development. Prog. Lipid Res. 2003, 42, 163-175. [CrossRef]

45. Wang, J.; Juliani, H.R.; Jespersen, D.; Huang, B. Differential profiles of membrane proteins, fatty acids, and sterols associated with genetic variations in heat tolerance for a perennial grass species, hard fescue (Festuca Trachyphylla). Environ. Exp. Bot. 2017, 140, 65-75. [CrossRef]

46. Lee, A.G. How lipids affect the activities of integral membrane proteins. Biochim. Biophys. Acta Biomembr. 2004, 1666, 62-87. [CrossRef]

47. Phillips, R.; Ursell, T.; Wiggins, P.; Sens, P. Emerging roles for lipids in shaping membrane-protein function. Nature 2009, 459, 379-385. [CrossRef]

(C) 2020 by the authors. Licensee MDPI, Basel, Switzerland. This article is an open access article distributed under the terms and conditions of the Creative Commons Attribution (CC BY) license (http://creativecommons.org/licenses/by/4.0/). 